\title{
Aplicação dos Gráficos de Controle CUSUM Tabular para Avaliação da Aderência dos Projetos ao Processo de Software
}

\author{
Paula Moreira ${ }^{1}$, Lenilda Pinheiro ${ }^{1}$, Jaciane Ribeiro ${ }^{2}$, Cleidson de Souza ${ }^{1}$, \\ Rodrigo Quites ${ }^{1}$ \\ ${ }^{1}$ Programa de Pós-Graduação em Ciência da Computação \\ (PPGCC-UFPA) Belém, PA - Brasil \\ ${ }^{2}$ Programa de Pós-Graduação em Matemática e Estatística \\ (PPGME-UFPA) Belém, PA - Brasil \\ \{pdanni,jacianedocarmo, lenilda\}@gmail.com, cdesouza@ufpa.br, \\ quites@computer.org
}

\begin{abstract}
This paper describes the experience of the authors in the usage of the technique CUSUM (Cumulative Sum) control charts to quantitatively evaluate software processes in a Level-2 CMM-SW certified Brazilian software development organization. This technique is used to evaluate to which extent the actual software processes of this organization match the prescribed software process.
\end{abstract}

Resumo. Este artigo descreve o uso do gráfico de controle CUSUM Tabular para avaliar quantitativamente a aderência dos projetos de software ao modelo de processo definido em uma organização de desenvolvimento de software certificada CMM-SW nível 2. Esta técnica é utilizada para avaliar aderência dos projetos ao processo de desenvolvimento de software.

\section{Introdução}

Em desenvolvimento de software, a qualidade pode ser entendida como um conjunto de características a serem satisfeitas de modo que o software atenda as necessidades explícitas e implícitas de seus usuários. Para avaliar a qualidade de um produto é necessário gerenciá-lo quantitativamente para obter medidas que quantifiquem o grau de uma característica da qualidade, sendo também necessária a utilização de um mecanismo para coleta e análise dessas medidas. Entretanto, a qualidade do produto depende fortemente da qualidade de seu processo de desenvolvimento (Fuggetta, 2000). Justamente por isso surgiram os modelos para avaliação dos processos de desenvolvimento de software. Estes estabelecem um conjunto de "melhores práticas" a serem seguidas para gerenciar o processo de desenvolvimento. Tais modelos surgiram a partir do sucesso de modelos de avaliação de processos propostos para indústria de manufatura. Dentre os principais modelos de avaliação de processo de software, destacam-se o CMMI (Chrissis et al, 2003) e o MPS.Br (SOFTEX, 2008).

Apesar dos recentes esforços das organizações brasileiras para adotar modelos de maturidade de processo de software, o que se observa hoje na maioria dessas organizações é uma grande quantidade de dados coletados sobre o processo, mas que 
não são utilizados para acompanhar o seu desempenho. Desta forma, não é possível determinar se os objetivos pretendidos pela organização estão sendo alcançados e se o processo de desenvolvimento é capaz de alcançá-los (Chrissis et al. 2003). Para dificultar ainda mais este quadro, os modelos de maturidade de processos de software, em seus níveis mais altos, exigem que o processo esteja sob controle estatístico, ou seja, que algum método estatístico seja utilizado para gerenciá-lo.

Segundo Ramos (2003), um método estatístico eficiente para acompanhar o desempenho de processos é o Controle Estatístico de Processo, ou simplesmente CEP, uma metodologia que envolve sete ferramentas estatísticas: Estratificação, Folha de Verificação, Diagrama de Ishikawa, Gráfico de Pareto, Histograma, Diagrama de Correlação e Gráficos de Controle. Dentre essas ferramentas, as mais difundidas e fáceis de interpretar são os gráficos de controle. Existem diferentes tipos de gráficos de controle que podem ser aplicados a processos, todavia, para engenharia de software, esses métodos são considerados relativamente inexplorados (Card, 2004). Pesquisadores como Cerdeiral et al (2007) e Florac (2000) têm testado a utilização de CEP em processos de software obtendo resultados bastante positivos. Entretanto, estes autores utilizam apenas os gráficos de controle de Shewhart para observações individuais $(\mathrm{XmR}$ ), para quantidade de não conformidades (gráfico $u$ ) ou para o gráfico das médias amostrais (X-bar). Estes gráficos têm baixa capacidade de detectar pequenas variações no processo (Montgomery, 2004) (Costa et al., 2005). Seguramente, melhorar o desempenho destes gráficos e/ou propor novas metodologias de construção e utilização dos mesmos, tornou-se um desafio para os pesquisadores e usuários do controle estatístico. Um problema comum identificado na utilização de gráficos de controle de Shewhart é o baixo poder que estes apresentam em detectar pequenas mudanças na característica de qualidade monitorada. Uma solução proposta para este problema é a utilização dos gráficos de controle CUSUM (Soma Acumulada) e/ou EWMA (Médias Móveis Ponderadas Exponencialmente), que são melhores em detectar pequenas mudanças no processo (Ramos, 2003), (Montgomery, 2004) (Costa et al., 2005). Dessa maneira, o objetivo deste trabalho é apresentar uma aplicação dos gráficos de controle CUSUM Tabular que permitem avaliar quantitativamente a estabilidade dos processos de desenvolvimento de software e que possam auxiliar a alta gerência na tomada de decisão. $\mathrm{O}$ artigo descreve a experiência dos autores ao aplicar os gráficos de controle CUSUM Tabular em uma organização de desenvolvimento de software federal certificada CMMSW nível 2, a regional Belém do SERPRO - Serviço Federal de Processamento de Dados.

O restante deste artigo está organizado como segue. A Seção 2 discorre brevemente sobre a área Garantia da Qualidade de Processo e Produto do CMMI (Capability Maturity Model Integration), enquanto que a Seção 3 descreve a metodologia Controle Estatístico de Processo. A Seção 4 apresenta o Estudo de Caso e a utilização do gráfico de controle CUSUM Tabular, enquanto que a Seção 5 descreve a análise e os resultados obtidos. Finalmente, a Seção 6 apresenta a conclusão do artigo e os trabalhos futuros vislumbrados. 


\section{Garantia da Qualidade do Processo e Produto}

Segundo Montoni (2006), a utilização dos resultados da área de processo da "Garantia da Qualidade do Processo e Produto de Software" tem sido cada vez mais adotada pela alta gerência das organizações. Os objetivos desta utilização são obter visibilidade quanto à qualidade dos processos executados e dos produtos de software entregues ao cliente, além de apoiar a tomada de decisões estratégicas do negócio.

Para garantir a correção dos resultados gerados por seus processos de negócios, algumas organizações baseiam-se nos modelos de maturidade de capacitação de referência internacional, tal como o CMMI, para definir seus processos. Neste modelo, existe a área de processo chamada "Garantia da Qualidade de Processo e Produto" cuja finalidade é fornecer à equipe e à gerência um entendimento objetivo dos processos e seus produtos de trabalho associados. Uma das metas desta área é avaliar quantitativamente os processos e produtos da organização. Tal avaliação é feita a partir de revisões que avaliam a aderência dos projetos de software às descrições de processo, padrões e procedimentos. Durante as quais, todos os artefatos elaborados são inspecionados por consultores da equipe de Garantia da Qualidade de Software (GQS) de acordo com checklists e experiência dos revisores designados para esta atividade. Os resultados das revisões podem revelar a presença de não conformidades nos artefatos. A metodologia utilizada para acompanhar o desempenho de processos de desenvolvimento de software será detalhada na seção a seguir.

\section{Controle Estatístico de Processo}

Para Montgomery (2004) o controle estatístico de processos é uma poderosa coleção de ferramentas utilizada para resolução de problemas úteis na obtenção da estabilidade do processo e na melhoria da sua capacidade por meio da redução de sua variabilidade. CEP é uma metodologia utilizada para avaliar se um processo encontra-se sob controle estatístico, ou seja, se ele possui desempenho, custo e qualidade previsíveis, além de auxiliar na melhoria contínua da qualidade e do desempenho do processo gerenciado (Florac, 1999).

Quando o processo não se encontra estável, devem-se estudar os pontos fora dos limites para determinar se as causas para tal fato são causas atribuíveis (especiais) ou causas comuns. As causas comuns traduzem as variações normais que ocorrem continuamente em qualquer processo, e são geradas pela interação entre os componentes do processo (pessoas, máquinas, ambiente, material, ambientes e métodos) sendo que são difíceis de ser controladas (Florac, 1999). As causas atribuíveis, entretanto, são geradas por alguma situação ou ação que pode justificar a variação ocorrida, sendo que tais causas podem ser controladas (Florac, 1999).

Dentre as ferramentas utilizadas no CEP, os gráficos de controle são mais utilizados devido à facilidade da sua aplicação e interpretação dos resultados. Desta forma, a seção a seguir detalha os gráficos de controle, identificando as características dessa ferramenta e enfatizando o tipo de gráfico de controle que será aplicado neste estudo.

\subsection{Gráficos de Controle}


Os gráficos de controle são definidos a partir de estatísticas (como a média, o desvio padrão amostral, etc.) e baseiam-se em certas distribuições de probabilidade, principalmente na distribuição normal. O gráfico de controle idealizado por Shewhart (Bravo, 1995) consiste simplesmente em três linhas paralelas: uma linha central, que reflete o nível de operação do processo, e duas linhas externas denominadas limite superior de controle $(L S C)$ e limite inferior de controle $(L I C)$. Os limites de controle são estabelecidos por três desvios-padrão $(\sigma)$, fazendo com que a probabilidade de um ponto estar dentro dos limites seja 99,74\%. Assim, no estado de estabilidade, a probabilidade de um ponto situar-se fora dos limites de controle torna-se muito pequena (Montgomery, 2004).

Quando há pontos além dos limites inferior e superior, considera-se o processo como instável, ou fora de controle estatístico, ou imprevisível. Fatos estes explicados pela presença de causas atribuíveis que atuam sobre o processo e que ocorrem de forma independente e sem controle. Todavia, quando todos os pontos estão distribuídos aleatoriamente, entre os limites inferior e superior, diz-se que o processo está estável, ou sob controle estatístico, ou ele é previsível. Assim, as variações se devem apenas por causas comuns. Por conseguinte, existem várias regras que explicam o comportamento do processo, que vão além da ocorrência de um valor excedendo os limites de controle.

\subsection{Gráfico de Controle CUSUM Tabular}

Segundo Bravo (1995), o gráfico de controle de somas acumuladas indica rapidamente a ocorrência de pequenas mudanças em um processo. Além disso, o gráfico de controle de somas acumuladas não está restrito a controle estatístico de processos, uma vez que eles podem ser usados para monitorar, por exemplo, o consumo de combustível em veículos de transporte, a resposta de pacientes a um tratamento médico ou o desempenho de um sistema de previsão.

Os gráficos CUSUM têm como principal característica detectar pequenas mudanças na distribuição da característica de qualidade e são aplicados tanto para observações individuais $(n=1)$ quanto para as médias das observações amostrais. No gráfico CUSUM, à medida que as amostras são retiradas, os desvios de $X_{i}$ em relação ao valor da média $\left(\mu_{0}\right)$ são acumulados, gerando a soma acumulada $C_{i}$, para o i-ésimo período, dada por:

$$
C_{i}=\sum_{i=1}^{n}\left(X_{i}-\mu_{0}\right)=\left(X_{i}-\mu_{0}\right)+C_{i-1}
$$

Os gráficos CUSUM não são considerados gráficos de controle por não apresentarem os limites de controle. Dentre as várias maneiras de representar os CUSUM's, destaca-se o gráfico CUSUM Tabular, o qual é considerado um gráfico de controle, pois estabelece seu limite de controle igual a $\mathrm{H}$ e supõe que os dados coletados seguem distribuição normal com média $\mu$ e desvio padrão $\sigma$. O gráfico de controle CUSUM Tabular utiliza as estatísticas $C^{+}{ }_{i}$ (detecta mudanças positivas) e $C_{i}{ }_{i}$ (detecta mudanças negativas) denominadas de CUSUM's unilaterais superior e inferior, respectivamente, e ambas são calculadas a partir das equações abaixo:

$$
C_{i}^{+}=\max \left[0, X_{i}-\left(\mu_{0}+K\right)+C_{i-1}^{+}\right] \quad \text { e } \quad C_{i}^{-}=\max \left[0,\left(\mu_{0}-K\right)-X_{i}+C_{i-1}^{-}\right]
$$


onde os valores iniciais $C^{+}{ }_{0}$ e $C_{0}^{-}$são iguais a zero; $X_{i}$ é a i-ésima observação amostral;

$\mu_{0}$ é o valor da média e $K=\left|\mu_{1}-\mu_{0}\right| / 2$ está relacionado à magnitude de mudança que se deseja detectar com o gráfico de controle de CUSUM. Como, observa-se, também, que $C^{+}{ }_{i}$ e $C_{i}^{-}$acumulam desvios em relação a $\mu_{0}$ e são igualados a zero quando se tornam negativos. $\mathrm{O}$ intervalo de decisão ou limite de controle utilizado no gráfico de controle CUSUM Tabular é $H$. Se o valor de $C^{+}{ }_{i}$ ou $C_{i}^{-}$exceder esse intervalo, pode-se dizer que o processo encontra-se fora de controle estatístico, logo, é preciso encontrar as causas atribuíveis que levaram o processo a tal estado e estimar seu valor médio para que se possa fazer o ajuste adequado (Alves, 2003). Geralmente, utiliza-se um valor para $H$ igual a cinco vezes o desvio padrão do processo $(H=5 \sigma)$. Portanto, no algoritmo do gráfico de controle CUSUM Tabular, existem os contadores denominados $N^{+}{ }_{i}$ e $N_{i}$ que indicam o número de períodos consecutivos em que os CUSUM's $C^{+}{ }_{i}$ e $C_{i}{ }_{i}$ assumem o valor zero. Costa et al. (2005) afirmam que esses indicadores são muito úteis para indicar o momento em que a média do processo alterou, sendo importante para auxiliar no diagnóstico dessa alteração. Para determinar o momento estimado da mudança, devese subtrair $N^{+}{ }_{i}$ ou $N_{i}$ do número da observação que sinalizou um estado fora de controle estatístico.

\section{Estudo de Caso}

A aplicação deste estudo de caso aconteceu em um pólo de desenvolvimento de software do SERPRO - Serviço Federal de Processamento de Dados - uma empresa pública de tecnologia da informação. Tal pólo foi certificado CMM-SW (Capability Maturity Model) nível 2 em dezembro de 2006 e possui três equipes com cerca de doze desenvolvedores cada. As amostras coletadas e utilizadas neste estudo referem-se às quantidades de não conformidades apontadas nos resultados de 128 revisões da Garantia da Qualidade de Software realizadas no pólo da Regional Belém no período de janeiro de 2005 a Fevereiro de 2008, ou seja, 38 observações normalmente distribuídas. Foram selecionados para a coleta 26 projetos de software de alta complexidade com esforço a partir de 150 homens-dia. Os períodos das coletas correspondem a três etapas de maturidades do pólo de software: a primeira de janeiro a dezembro de 2005 onde ocorreram estudos, treinamentos e disseminação do CMM-SW nível 2; a segunda etapa, de janeiro a dezembro de 2006, período onde ocorreram as avaliações corporativas do nível de maturidade do processo e a terceira etapa de janeiro de 2007 a fevereiro de 2008, período posterior a certificação CMM-SW nível 2. As quantidades de não conformidades são apontadas durante as revisões GQS, cujo objetivo é inspecionar o nível de aderência dos artefatos elaborados/atualizados durante a execução dos projetos de software ao Processo Serpro de Desenvolvimento de Soluções (PSDS), um modelo de processo institucionalizado na organização.

Para que as revisões sejam realizadas, um Plano de GQS é elaborado pelo consultor GQS designado, no qual são planejadas todas as revisões GQS até o encerramento do projeto e o mesmo é elaborado baseando-se no plano de projeto, planejamento de esforço, custo, prazo e cronograma do projeto. Todas as revisões dos artefatos de software são cadastradas em um sistema chamado Revisa. Este sistema é responsável pelo agendamento das revisões e notificações das partes interessadas, além 
de permitir o armazenamento dos resultados das revisões, acompanhamento das correções das não conformidades e disponibilização de relatórios e consultas gerenciais. As revisões previstas são realizadas mensalmente. As não conformidades encontradas são reportadas às equipes para correção e tomada de ação para melhoria da aderência do projeto ao processo.

A partir da coleta das quantidades de não conformidades armazenadas no sistema Revisa aplicou-se o gráfico de controle CUSUM Tabular para observações individuais visando acompanhar se os projetos de software estão aderentes ao processo definido pela organização. A Figura 1 apresenta as estatísticas CUSUM Superior $(\mathrm{C}+\mathrm{i})$ e CUSUM Inferior (C-i) para a quantidade de não conformidades apontadas nos resultados das revisões GQS no período de janeiro de 2005 a fevereiro de 2008. O limite superior $\mathrm{H}$ igual a $5 \sigma$ foi considerado, pois este é um valor condizente com a literatura da área. Como o valor do desvio padrão foi 6,25, o valor do limite superior H foi 31,25.

Observa-se que com relação aos valores $\mathrm{C}^{+}$, que a observação referente ao mês de maio de 2006 (observação 17), encontra-se acima do limite superior de controle $(L S C=31,25), \operatorname{com} N^{+}{ }_{i}=17(17-17=0)$. Com isso, estima-se que o aumento das não conformidades inicia desde janeiro de 2005 (observação 1), pois a subtração do número da observação que sinalizou o aumento de $N^{+}{ }_{i}$ indica o momento estimado da mudança. Isso é importante para que as causas atribuíveis sejam identificadas e a organização possa tomar decisões baseada não somente nos fatos ocorridos no período em que a observação referente ao mês de maio de 2006 excedeu os limites, mas que uma análise mais detalhada possa ser realizada, a partir do período de início da variação.

Com relação aos valores do CUSUM Inferior $\left(\mathrm{C}_{i}^{-}\right)$, observa-se que a partir da observação referente ao mês de junho de 2007 (observação 30) a quantidade de não conformidades nas revisões GQS encontra-se acima do limite superior de controle, com $\mathrm{N}_{i}^{-}=13(30-13=17)$. Logo, estima-se que essa redução inicia entre maio de 2006 (observação 17) e junho de 2006 (observação 18), pois a subtração do número da observação que sinalizou a diminuição de $\mathrm{N}^{-}$indica o momento estimado da mudança.

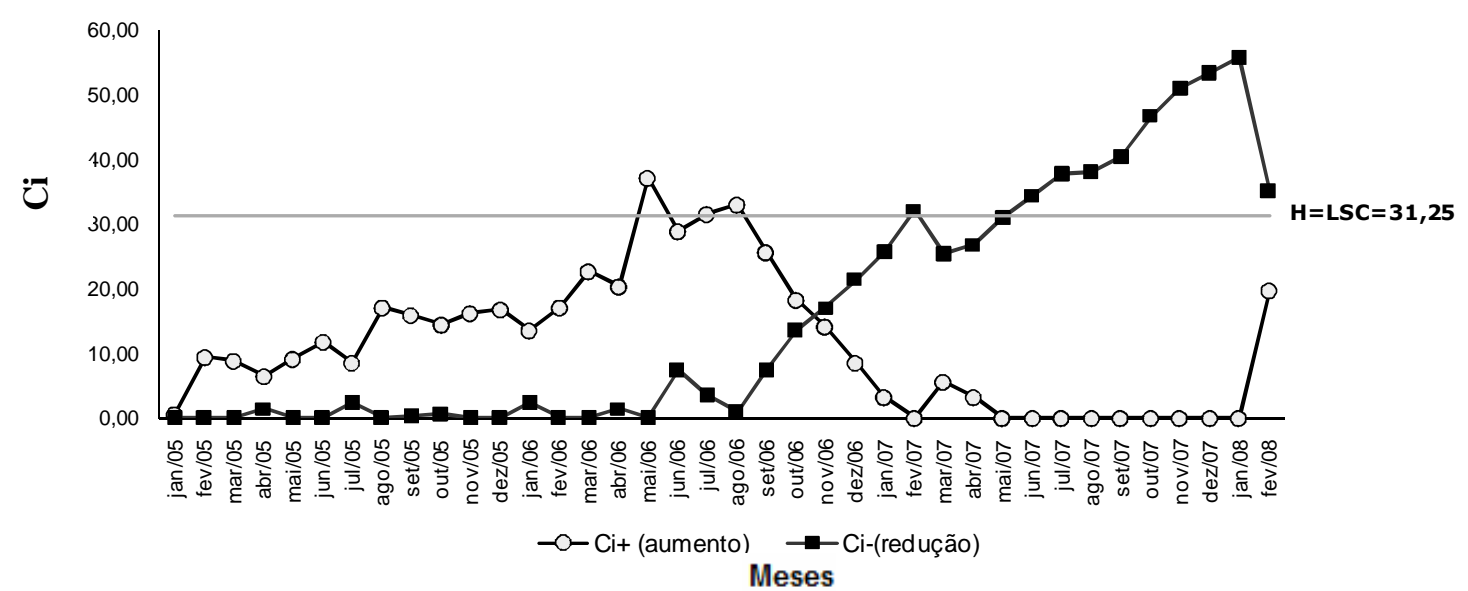

Figura 1. Gráfico de Controle CUSUM Tabular para a quantidade de Não Conformidades Apontadas nos Resultados das Revisões GQS no Período de janeiro de 2005 a fevereiro de 2008. 


\section{Análise e Interpretação dos Resultados}

A partir do gráfico de controle CUSUM Tabular, observa-se um aumento na quantidade de não conformidades de janeiro de 2005 a maio de 2006. Entrevistas com os envolvidos na gerência das equipes de GQS foram realizadas e constatou-se que uma possível razão para o aumento na quantidade de não conformidades nesse período foi que a equipe de desenvolvimento estava em fase de aprendizado, portanto, os artefatos não eram bem elaborados/atualizados. Além disso, novas pessoas foram admitidas na organização, o que consequientemente trouxe dificuldade para os novos integrantes em seguir o processo de desenvolvimento utilizado pela empresa. Assim como os revisores adquiriam mais experiência a partir das medidas adotadas pela organização para melhorar a revisão (treinamentos, mentorias, etc.), os desenvolvedores e líderes de projetos também evoluíram na elaboração/atualização dos artefatos, uma vez que a organização estava investindo na certificação, o que motivou os desenvolvedores e gerentes a seguirem o processo definido. Com estas medidas, a quantidade de não conformidades diminuiu e a partir do gráfico CUSUM Tabular, observa-se que essa diminuição começa entre os meses de maio e junho de 2006.

Uma observação importante foi que os projetos tiveram um aumento da aderência ao processo definido, já que a quantidade de não conformidades diminuiu. Em Dezembro de 2006, a organização conseguiu a certificação CMM-SW nível 2, quando a avaliação foi realizada, acreditava-se que os projetos estavam sendo seguidos de acordo com o estabelecido no processo, contudo, não se tinha uma forma de confirmar tal fato. Com a utilização da abordagem sugerida pôde-se perceber que a certificação poderia ter sido alcançada antes do período, pois desde junho de 2006 os projetos estavam mais aderentes ao processo.

\section{Conclusão}

Este trabalho está alinhado com a noção do uso de técnicas estatísticas baseadas em controle de processo para avaliação e melhoria da qualidade de software. Deve-se ressaltar que a adoção destas técnicas tem crescido recentemente (Silva, 2005). O trabalho aqui apresentado trouxe resultados significativos, e destaca-se por permitir que a organização estabeleça ações corretivas ou adaptativas, a partir dos problemas identificados pelo gráfico de controle e, também, acompanhar se estas ações asseguraram melhores resultados à aderência do processo definido. Vale ressaltar que a os gráficos de controle padrões de Shewhart não conseguem mostrar desde quando o processo está fora de controle, o que dificulta as análises das possíveis causas de variação no processo. Além disso, a aplicação do CEP nas fases iniciais de definição dos processos de software tem o objetivo de identificar precocemente quais áreas do processo podem ser melhoradas, além de proporcionar a possibilidade de acompanhar estatisticamente o processo em suas fases iniciais, o que é uma das exigências estabelecidas pelos modelos de avaliação de processo em seus níveis mais altos.

Alguns aspectos podem ser observados como trabalhos futuros, tais como: aplicação da técnica em outras áreas do processo, como o teste de software para que se consiga acompanhar o desempenho desta atividade e se possa encontrar problemas e procurar soluções para os mesmos e que as lições aprendidas pela empresa possam ser 
compartilhadas. O nível de aderência ao processo pode ser medido também pelo índice de capacidade do processo (IC) que é utilizado quando os gráficos estão sob controle estatístico, ou seja, quando nenhuma causa atribuível ocorre. Em outras palavras, o IC ajuda a mensurar a capacidade do processo. De um modo geral, nos trabalhos futuros pretende-se utilizar os gráficos de controle com dois objetivos: monitorar o desempenho do processo na busca de causas de instabilidade e, caso o processo esteja sob controle, avaliar a capacidade do processo para verificar se as necessidades da organização foram alcançadas.

\section{Agradecimentos}

Ao SERPRO, por autorizar a publicação dos dados e resultados do estudo realizado.

\section{Referências}

Alves, Custódio C. Gráficos de Controle CUSUM: um enfoque dinâmico para a análise estatística de processos. 2003. Dissertação (Mestrado em Engenharia de Produção) PPGEP/UFSC, Florianópolis, Santa Catarina, Brasil.

Bravo, P. C. Controle Estatístico da Qualidade, In: 40a Reunião Anual da Região Brasileira da Sociedade Internacional de Biometria (RBRAS), Ribeirão Preto - São Paulo, 18- 21/07/1995. Anais da 40ª RBRAS e $6^{\circ}$ SEAGRO, Ribeirão Preto, 1995.

Card, D.N. Statistical Techniques for Software Engineering Practice. Software Productivity Consortium. Proceedings of the 26th International Conference on Software Engineering, 2004.

Cerdeiral, C., Figueiredo, S., Santos, G. Montoni, M. Pinto, R. Rocha, A. C. Uma Abordagem para Controle Estatístico do Processo e Gerência Quantitativa de Projetos. Simpósio Brasileiro de Qualidade de Software (SBQS), 2007.

Chrissis, M. B., Konrad, M., Shrum, S. CMMI: Guidelines for Process Integration and Product Improvement, Addison Wesley, 2003.

Costa, A. F.; Epprecht, E. K.; Carpinetti, L. C. R. Controle Estatístico de Qualidade. Atlas, 2005, 2a Edição.

Florac, A. W., C., Barnard, A. D. Measuring the Software Process: Statistical Process Control for Software Process Improvement. Addison Wesley, 1999.

Florac, A.W., C., Barnard, A.D., , J.R. Statistical Process Control: Analyzing a Space Shuttle Onboard Software Process, IEEE Software, pp. 97-106, 2000.

Fuggetta, A. Software Processes: A Roadmap. Future of Software Engineering, Limerick, Ireland, 2000.

Montgomery, D. Introdução ao Controle Estatístico da Qualidade. 4a. Ed. Rio de Janeiro: LTC, 2004.

Montoni, M., Santos, G., Figueiredo, S., Cabral, R., Barcelos, R., Barreto, A., Barreto, A., Cerdeiral, C., Lupo, P., Rocha, A. R.. Uma Abordagem de Garantia de Qualidade de Processos e Produtos de Software com Apoio de Gerência de 
Conhecimento na Estação TABA. V Simpósio Brasileiro de Qualidade de Software (SBQS'06). Vila Velha, Brasil, 2006.

Ramos, E. M. L. S. Aperfeiçoamento e Desenvolvimento de Ferramentas do Controle Estatístico da Qualidade utilizando Quartis para estimar o Desvio Padrão. Tese de Doutorado, Universidade Federal de Santa Catarina, Abril, 2003.

Silva, R. C. F., Rocha, A. R. C., Travassos, G. H. Uma Abordagem Experimental para a Avaliação da Melhoria de Processos. IV Simpósio Brasileiro de Qualidade de Software (SBQS'05). Porto Alegre, Brasil, 2005.

SOFTEX - Associação para Promoção da Excelência do Software Brasileiro. MPS.BR - Guia Geral, v1.2, 2008. Disponível em www.softex.br. 